\title{
A robust region_based active contours model for image segmentation
}

\author{
Jiandong Bao \\ (School of Mechanical Engineering, Nanjing \\ University of Science and Technology) \\ (Nanjing, China) \\ (e-mail: 13913876328@163.com)
}

\author{
Fan Jiang \\ (School of Mechanic al Engineering, Nanjing \\ University of Science and Technology) \\ (Nanjing, China) \\ (e-mail:441338195@qq.com)
}

\begin{abstract}
A novel region_based active contours model is proposed to deal with the images with intensity inhomogeneities and weak boundaries. For the proposed model, new global force and local force which compose hybrid energy functional are defined. Then, the energy functional is incorporated into a variational level set formulation. Furthermore, we regularize the level set function by using Gaussian filtering to keep it smooth and eliminate the re-initialization. In addition, the proposed model can degrade to a new global $\mathrm{CV}$ model. Experiments results show that the proposed model can not only segment images with intensity inhomogeneities and weak boundaries, but also robust to the noise, initial contours. And, it has high computational efficiency.
\end{abstract}

Keywords - Image segmentation; Active contours model; Intensity inhomogeneities; Initial contours; Noise

\section{I . INTRODUCTION}

Image segmentation has been a research hotspot and difficulty in image processing and machine vision. Based on active contour model is applied in the field of image segmentation in recent years is a successful method. Active contour model can be divided into active contour based on edge ${ }^{[1-2]}$ and the regional $\operatorname{model}^{[3-4]}$.

Based on the edge of the active contour model use gradient information of image, Driving the contour curve to stop at the edge of the target, such as geodesic active contour model ${ }^{[2]}$.However, when the target edge blur or uneven gray, This method will not be able to segment the target correctly. Area based active contour with the image of local information (such as gray scale, texture) to guide the contour curve of the boundary approximation to the target object. One of the most famous is the $\mathrm{CV}$ model $^{[3]}$, The CV model is based on the as sumption of the gray of internal and external contour curves are uniform, so when appear uneven gray-level, the CV model will not be effective segmentation. As a result, some of the active contour model based on local statistical information was put forward ${ }^{[4-9]}$.For example, $\mathrm{Li}^{[4,5]}$ introduce the local information to $\mathrm{CV}$ model, namely the RSF model. The model in the uneven illumination of the image segmentation is useful. Zhang ${ }^{[6]}$ put forward the LIF model, the model and RSF model have the same segmentation effect, but greatly improve the computational efficiency. However, these methods due to using only local information are sensitive to the initial curve, easy to fall into local minimum. Aiming at these problems, some combination of global and local energy combined methods have been proposed ${ }^{[10-11]}$. Such method has the advantages of the global methods and local methods at the same time, the boundary image was able to handle non homogeneity and has a good robustness to weak initial curve. However, this kind of approach has led to some new problems, For example, a double border ${ }^{[11]}$, Cannot deal with the image which has noise effectively ${ }^{[10]}$ and so on.

In the view of the problems, this paper presents a new robust active contour model. First of all, using statistics information of images to build a new global and local force. Second, using the weighted combination of these two kinds of force to get a mix of energy function. Level set method is used to minimize the energy functional, level set evolution 
equation is obtained and updated constantly. Finally, by adopting the method of Gaussian filtering rules to the level set equation. Synthetic images and real images of the experimental results show that this model can effectively segment the homogeneity or weak edge image, has a good robustness to the noise as well as the initial contour curve, and high calculation efficiency.

\section{MODEL TO COMPARE}

\section{A. CV Model}

The energy function of $\mathrm{CV} \operatorname{model}^{[3]}$ which is defined as follows:

$$
\begin{aligned}
& E^{C V}\left(c_{1}, c_{2}, C\right)=\int_{\text {inside }(C)}\left(I-c_{1}\right)^{2} d x+\int_{\text {outside }(C)}\left(I-c_{2}\right)^{2} d x+v|C| \\
& +\mu \cdot \operatorname{Area}(\text { inside }(C))
\end{aligned}
$$

In the formula, inside( $C)$ and outside(C) represent the internal and external of curve $\mathrm{C}, c_{1}$ and $c_{2}$ separately represent the curve of average gray value of the internal and external.

Formula in the third and that in the fourth are the length and area inside of the curve. The formula (1), expressed as the zero level set evolution curve, the corresponding level set equation is:

$$
\frac{\partial \phi}{\partial t}=\delta_{\varepsilon}(\phi)\left[v \operatorname{div}\left(\frac{\nabla \phi}{|\nabla \phi|}\right)-\mu-\left(I-c_{1}\right)^{2}+\left(I-c_{2}\right)^{2}\right]
$$

In the formula:

$$
\left\{\begin{array}{c}
c_{1}=\frac{\int_{\Omega} I(x) \cdot H_{\varepsilon}(\phi) \mathrm{d} x}{\int_{\Omega} H_{\varepsilon}(\phi) \mathrm{d} x} \\
c_{2}=\frac{\int_{\Omega} I(x) \cdot\left(1-H_{\varepsilon}(\phi)\right) \mathrm{d} x}{\int_{\Omega}\left(1-H_{\varepsilon}(\phi)\right) \mathrm{d} x}
\end{array}\right.
$$

$H_{\varepsilon}(\phi)$ and $\delta_{\varepsilon}(\phi)$ are Heaviside function and Dirac function, are defined as follows:

$$
\left\{\begin{array}{c}
H_{\varepsilon}(\phi)=\frac{1}{2}\left[1+\frac{2}{\pi} \arctan \left(\frac{\phi}{\varepsilon}\right)\right] \\
\delta_{\varepsilon}(\phi)=\frac{1}{\pi} \frac{\varepsilon}{\varepsilon^{2}+\phi^{2}}
\end{array}\right.
$$

In this paper, for the simple, still puts them abbreviated as $H(\phi)$ and $\delta(\phi)$ respectively below. Due to $c_{1}$ and $c_{2}$, respectively are the gray scale average curve of internal and external, are a global, makes the model of initial curve and noise a good robustness, but because this model does not include local information, when the energy functional to the minimum, contour curve deviates from the target boundary and so that it cannot be accurately even gray-level image segmentation.

\section{B. LRCVModel ${ }^{[7]}$}

Liu set an active contour model which is improved, written for the LRCV model ${ }^{[7]}$. It is different from the CV model which defines pixels and global average difference energy function, the model adopts the neighborhood pixels and the pixels difference for powering fitting, similar to the model of the RSF. And the adoption of the Gaussian filter finally made the model a good robustness for noise and smoothness. The energy function of the model is as follows:

$$
\begin{aligned}
& E\left(f_{1}, f_{2}, \phi\right)=\lambda_{1} \int_{\Omega}\left(I(x)-f_{1}(x)\right)^{2} H(\phi(x)) d x \\
& +\lambda_{2} \int_{\Omega}\left(I(x)-f_{2}(x)\right)(1-H(\phi(x))) d x
\end{aligned}
$$

In this formula, $f_{1}$ and $f_{2}$ represent the weighted average of the mean value of the curve, this is the area of inside and outside, defined as:

$$
\left\{\begin{array}{c}
f_{1}(x)=\frac{K_{\sigma} *[H(\phi) I(x)]}{K_{\sigma} * H(\phi)} \\
f_{2}(x)=\frac{K_{\sigma} *[(1-H(\phi)) I(x)]}{K_{\sigma} *(1-H(\phi))}
\end{array}\right.
$$

This method computes the image gray level of each pixel point and its neighborhood weighted difference of gray value to build the energy function. This model can deal with gray uneven and weak edge image effectively. Due to the model only using the local characteristics and ignoring the global information, which makes it sensitive to initial contour curve and noise. 


\section{The optimization model}

Comprehensive CV model combining initial curve with good noise robustness and the noise resistance and smoothness of LRCV model, this paper proposes a combination of global and local information of the mixed area of Active contour model (Hybrid region_based Active Contours, HRAC), the energy function is defined as follows:

$$
\mathrm{E}=\alpha \mathrm{E}^{1 \mathrm{o} \mathrm{b}}+(1-\alpha) \mathrm{E}^{1 \mathrm{oc}}
$$

Among them, $\alpha \in[0,1]$ to weigh the weight of global and local forces, when uneven gray level is serious, you should fit small value to make the local force primary.

Assumes that the curve, both inside and outside gray are uniform, the fitting image is defined as follows.

$$
I^{G F I}(x)=c_{1}(x) \cdot H(\phi)+c_{2}(x) \cdot(1-H(\phi))
$$

For $c_{1}(x)$ and $c_{2}(x)$ are interior and exterior grayscale of curve, when the curve is located in the target boundary, the energy function of the following will be minimized:

$$
\mathrm{E}^{\text {global }}=\frac{1}{2}\left(I(x)-I^{G F I}(x)\right)^{2}
$$

Local models in this paper adopt the LRCV model ${ }^{[7]} \phi$ is this article's hybrid energy on the level set functional:

$$
\begin{aligned}
\mathrm{E} & =(1-\alpha) \cdot\left(\int_{\Omega}\left(I(x)-f_{1}(x)\right)^{2} H(\phi(x)) d x+\int_{\Omega}\left(I(x)-f_{2}(x)\right)(1-H(\phi(x)))\right) \\
& +\frac{1}{2} \alpha \cdot\left(I(x)-I^{G F I}(x)\right)^{2}
\end{aligned}
$$

Using gradient descent flow ${ }^{[12]}$ to minimize $\phi$ values of E, geometric flow can be calculated as follows:

$$
\begin{aligned}
\frac{\partial \phi(x, t)}{\partial t} & =\delta(\phi) \cdot\left[((1-\alpha))\left(-\left(I(x)-f_{1}(x)\right)^{2}+\left(I(x)-f_{2}(x)\right)^{2}\right)\right] \\
& +\delta(\phi) \cdot \alpha \cdot\left(I(x)-I^{G F I}(x)\right)\left(c_{1}-c_{2}\right)
\end{aligned}
$$

of level set evolution in the process, this article continues to use the LRCV model of Gaussian convolution methods to smooth the level set function, so as to avoid the calculation of costly reinitializations $^{[3]}$ and regularizations ${ }^{[2,5,6]}$.

$$
\phi^{n+1}=G_{\varsigma} * \phi^{n}
$$

Among them, $S$ is the variance of Gaussian convolution, Gaussian convolution size is $k \times k, \mathrm{n}$ is the number of iterations.

In conclusion, in this paper, the algorithm implementation steps are as follows:

Step1 initialize level set function $\phi$ to binary function;

Step2 calculate $c_{1} 、 c_{2} 、 f_{1} 、 f_{2}$;

Step3 according to (11), update the level set function;

Step4 according to (12) through a Gaussian filter smooth level set function;

Step5 determine $\phi$ is convergence or not, if no convergence, return to step 2 .

\section{THE EXPERIMENTAL RESULTS AND ANALYSIS}

This section will verify optimization model in the paper: (1) compared with the CV model, the optimization model can segment uneven gray-level and edge blur image; (2) compared with the LRCV model, the initial contour curve of optimization model has better robustness; (3) compared with the model CVRSF in reference [10], the noise of optimization model has a better robustness and higher computation efficiency.

The entire experimental environ ment is Intel (R) Core (TM) Duo CPU 2.00 GB of PC, MATLAB R2010a. The model of the default parameter settings are as follows: $\alpha=0.01, \Delta t=0.025$, and the span of $\varsigma$ is $[1,3]$, the span of $k$ is $[3,13]$.

The experimental comparison in figure 1 is the optimization model and CV model, which show a group of uneven illu mination ' $\mathrm{T}$ ' vascular image and synthetic image. The sizes of the image are $103 \times 131$ and $127 \times 96$. Blood vessels of $\mathrm{X}$-ray image and ' $\mathrm{T}$ ' image both demonstrate gray distribution and the target edge of blood vessels is blur. According to the experimental results, compared with the CV model, this model can distinguish uneven illumination and the weak edge images. 

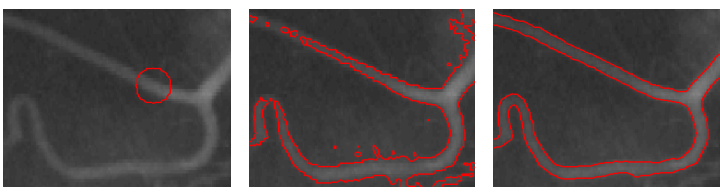

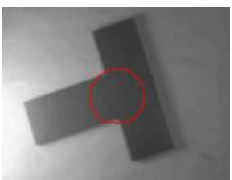

(a) initial curve

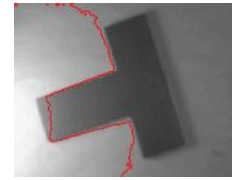

(b) CV Model

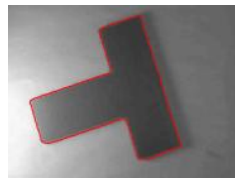

(c) optimization model
Figure.1 Segmentation results using the CV model and the propose d model

The experiments compare the in itial curve on the proposed model and the LRCV model ${ }^{[7]}$ as in figure (2). The image sizes are $111 \times 110$ and $127 \times 96$. The initial contour curve radius of blood vessel image experiment is 10 , which chooses different position of initial. It can be seen that proposed model is not affected by the influence of in itial contour curve and it can segment the target successfully, while the LRCV model only in some special place can get correct segmentation result. The initial contour curve of ' $T$ ' image experiment radius size is 15 , which picks different position of in itial. As the figure shown, the LRCV model segmentation results are relatively dependent on the initial curve, namely when choosing suitable initial curve, the model can segment the target LRCV model, it can obtain the ideal segmentation results. When the proposed model segment weak edges and non homogeneous image, it has a good robustness to initial contour curve.
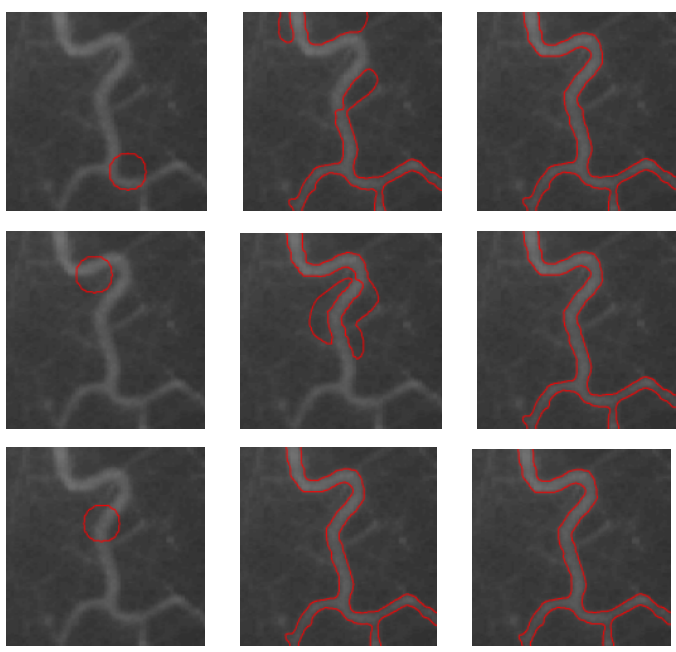

(a) initial curve

(b)LRCV Model
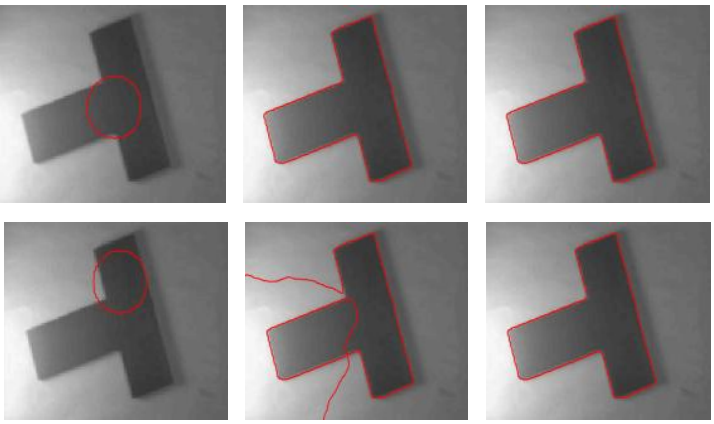

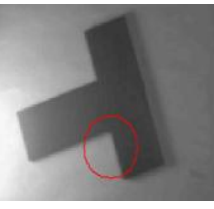

(a) initial curve

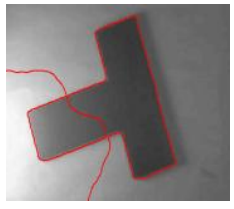

(b)LRCV Model

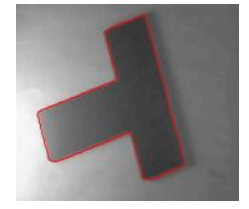

(c)optimization model
Figure. 2 Segmentation resul ts using the $\mathrm{CV}$ model, the LRCV model and the proposed model for the vessel images and ' $T$ ' image

Experiments in figure 3 is to prove the high computational efficiency, using literature in [10] CVRSF model and the model in this paper. The initial curves of two models are the same, and to compare the efficiency of the two algorithms, set their parameters are the same. Table 1 shows that when the two models get same segmentation results, the number of iteration and the calculation time they separately needed.
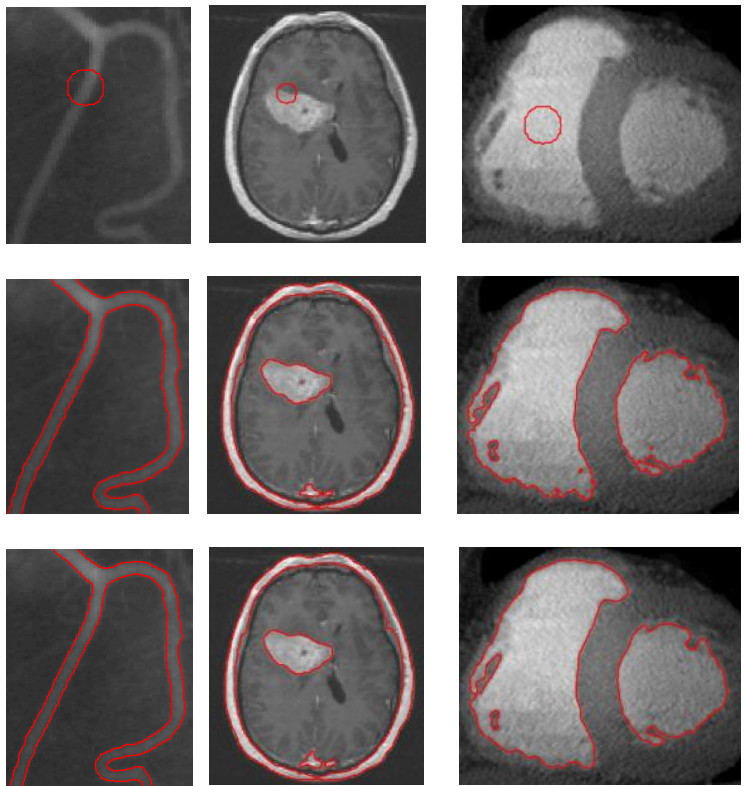

Figure. 3 Segmentation resul ts using the CVRSF model and the propose d model (Row 1: Initial con tours; Row 2: Results of the CVRSF model; Row 3: Results of the propose dmodel) 
TABLE I . THE ITERATIONS AND THE PROCESSING TIME OF THE C VRSF MODEL AND THE PROPOSED MODEL

\begin{tabular}{|c|c|c|c|c|c|c|}
\hline & \multicolumn{2}{|c|}{ Vessel } & \multicolumn{2}{c|}{ brain image } & \multicolumn{2}{c|}{ Heart image } \\
\hline & $\begin{array}{c}\text { Itera } \\
\text { tions }\end{array}$ & $\begin{array}{c}\text { Time } \\
(s)\end{array}$ & $\begin{array}{c}\text { Itera } \\
\text { tions }\end{array}$ & $\begin{array}{c}\text { Time } \\
(s)\end{array}$ & $\begin{array}{c}\text { Itera } \\
\text { tions }\end{array}$ & $\begin{array}{c}\text { Time } \\
(s)\end{array}$ \\
\hline CVRSF & 180 & 19.5 & 70 & 20.56 & 30 & 11.46 \\
\hline $\begin{array}{c}\text { optimization } \\
\text { model }\end{array}$ & 100 & 8.95 & 40 & 9.23 & 10 & 2.36 \\
\hline
\end{tabular}

According to the table 1 , because this article model abandons a rule which maintains smooth rules and it uses a Gauss filter method to keep smooth. When gets the same segmentation result, this algorithm needs less iterative number and time compared with others. Experiment proved that this model compared with CVRSF model has higher computing efficiency.

The experiment of figure 4 is the comparis on of CVRSF model and the optimization model on noise resistance. Add zero mean Gaussian noise to the synthesis of uneven gray-level images, Gaussian noise variance adding from left to right in turn was $0.001,0.01,0.04$. When the noise variance increases gradually, CVRSF will failure completely, and this model can also get the boundary of the target when the goals in the noise. The experimental results show that compared with CVRSF model, this model has good robustness for noise.
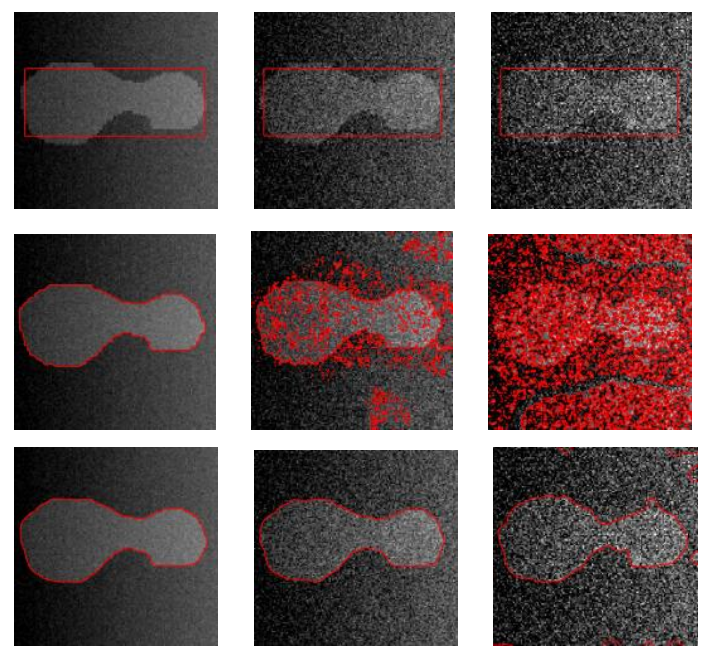

Figure. 3 Segmenting noisy images with different variances

using the CVRSF model and the propose dmodel (Rows 1, 2

and 3 are added Gaussian white noise with variances 0.001 ,

0.01, and 0.04, respectively.) Row 2: Results of the CVRSF model; Row 3: Results of the proposed model)

\section{CONCLUSION}

This paper presents a new robust regional active contour model. First of all, for the proposed model, new global force and local force which compose hybrid energy functional are defined. Then, the energy functional is incorporated into a variation level set formulation. Furthermore, we regularize the level set function by using Gaussian filtering to keep it smooth and eliminate the re-initialization. In addition, the proposed model can degrade to a new global CV model. Experiments results show that the proposed model can not only segment images with intensity in homogeneities and weak boundaries, but also robust to the noise, initial contours. And, it has high computational efficiency.

\section{REFERENCES}

[1] V. Caselles, R. Kimmel, G. Sapiro. Geodesic active contours[J]. International Journal of Computer Vision.1997, 22(1): 61-79

[2] Chunming Li, Chenyang $\mathrm{Xu}$, Changfeng Gui et al. Level set formulation without Re-initialization: a new variational formulation $[J]$. Proceedings of the IEEE International Conference on Computer Vision and Pattern Recognition (CVPR) 2005, 2005, 1(1): 430-436.

[3] T. Chan and L.Vese. Active contours without edges[J]. IEEE Trans. Imag. Proc., 2001, 10:266-277.

[4] Chunming Li, Chiu-Yen Kao, John C. Gore, et al. Minimization of region-scalable fitting energy for image segmentation[J]. IEEE Transactions on Image Processing, 2008, 17: 1940-1949.

[5] Chunming Li, Chiu-Yen Kao, John C. Gore et al. Implicit active contours driven by local binary fitting energy[A]. IEEE Conference on Computer Vision and Pattern Recognition[C], 2007:17-22.

[6] Kaihua Zhang, HuihuiSong, LeiZhang. Active contours driven by local image fitting energy[J]. Pattern Recognition, 2010, 43: 1199-1206.

[7] Shigang Liu, Yali Peng. Peng. A local region-based Chan-Vese model for image segmentation[J]. Pattern Recognition, 2012, 45: 2769-2779.

[8] Yang Mingyu, Ding Huan, Zhao Bo et al. Chan-Vese Model Image Segmentation with Neighborhood Information [J]. Journal of Computer-Aided Design\& Computer Graphics, 2011, 23(3): 413-418. (in Chinese)

[9] Li Wang, LeiHe, ArabindaMishra et al. Active contours driven by local Gaussian distribution fitting energy[J]. Signal Processing, 2009, 89:2435-2447.

[10] Li Wang, Chunming Li, Quansen Sun, Deshen Xiaa, et a1. Active contours driven by local and global intensity fitting energy with application to brain MR image segmentation[J]. Computerized Medical Imaging and Graphics, 2009, 33(7): 520-531.

[11] Xiao-Feng Wang, De-ShuangHuang, HuanXu. An efficient local Chan-Vese model for image segmentation[J]. Pattern Recognition, 2010, 43:603-618.

[12] L. Evans Partial Differential Equations[M]. Providence: American Mathematical Society, 1998. 